\title{
Relativistic Adiabatic Shocks in Accretion Flows
}

\author{
D. M. Caditz and S. Tsuruta \\ Department of Physics, Montana State University, Bozeman, MT \\ 59717, U.S.A.
}

\begin{abstract}
Accretion flows onto compact astronomical sources are likely to be supersonic, and shock waves may therefore be common in such flows. Plasma passing through a shock front will be compressed and heated according to the jump conditions across the shock discontinuity. Shocks in accretion flows may therefore have important consequences for the flow structure and emission characteristics. The equations governing adiabatic (nonradiative) shocks in relativistic plasmas are presented including the effects of radiation pressure and energy density, and pair equilibria in the postshock flow. We find that postshock states for accretion flows within cool, optically thick, accretion-driven sources such as AGN become radiation- or pair-dominated, and the postshock plasma will likely become optically thin before returning to steady-state conditions.
\end{abstract}

\section{Introduction}

In a wide range of astronomical plasmas, the conditions appropriate for shocks may be present. Shocks may be an important feature of, e.g., accretion flows, winds, jets, supernovae, star formation, stellar flares, et cetera, and radiation from hot postshock material may be identified in such sources.

Shock models relating pre- and postshock conditions rely on 1) conservation conditions across the shock front and 2) the bulk thermodynamic properties of the shocked plasma. Common treatments that invoke nonrelativistic conservation laws and polytropic equations of state are not accurate under relativistic conditions expected in a wide range of astronomical sources.

In this poster, we present the results of relativistically correct shock calculations using realistic equations of state for the pre- and postshock plasma applicable to accretion flows onto compact objects. In addition, we investigate possible dynamics and radiative effects of the heated postshock plasma. We find that shocked plasma will likely become optically thin and therefore lose excess energy in a flare or burst of high-energy radiation. The results presented here are expected to apply to AGNs and accretion-powered galactic X-ray sources.

\section{Conservation Equation}

The Taub adiabat relates pre- and postshock thermodynamic states for adiabatic shocks (Iwamoto 1989): 


$$
\frac{w_{1}^{2}}{n_{1}^{2}}-\frac{w_{2}^{2}}{n_{2}^{2}}+\left(P_{2}-P_{1}\right)\left[\frac{w_{1}}{n_{1}^{2}}+\frac{w_{2}}{n_{2}^{2}}\right]=0 .
$$

Here, the enthalpy density $(w)$, pressure $(P)$, and baryon number density (n) are to be expressed in the respective fluid-element rest frames, and the subscripts 1 and 2 refer to the pre- and postshock conditions, respectively. The nonrelativistic version of this equation is the well-known Rankine-Hugoniot equation for classical adiabatic shocks.

\subsection{State Equation}

Shock adiabatics are obtained by inserting the appropriate state functions, that is to say, $w_{i}\left(P_{i}, n_{i}\right)$, into Eq. (1). We consider a general equation of state including radiation and pair effects:

$$
P=P_{g a s}+P_{r a d}=n m_{e} c^{2}(2+2 z) q+\frac{1}{3} a q^{4}
$$

and

$$
\begin{aligned}
\varepsilon & =\varepsilon_{\text {protons }}+\varepsilon_{\text {electrons }}+\varepsilon_{\text {rad }} \\
& =n m_{p} c^{2}\left(1+\frac{3}{2} \frac{m_{e}}{m_{p}} q\right)+n(1+2 z) F(1 / q)+a q^{4} .
\end{aligned}
$$

Here, the variables are $q=k_{b} T /\left(m_{e} c^{2}\right), z \equiv n_{+} / n_{p}$ is the pair to proton ratio, $a=4 \sigma / c\left(m_{e} c^{2} / k_{b}\right)^{4}=9.42 \times 10^{24} \mathrm{erg} \mathrm{cm}^{-3}$, and $F(x)=m_{e} c^{2} \frac{3 K_{3}(x)+K_{1}(x)}{4 K_{2}(x)}$ where $K_{\nu}$ is the modified Bessel function of the second kind of order $\nu$.

The pair density ratio is discussed by Svensson (1984), Caditz \& Tsuruta (1998), and references therein.

\subsection{Shock Adiabats}

The above equations allow us to calculate shock adiabats, $P(1 / n)$, for given initial conditions, $\left(P_{1}, n_{1}\right)$ or, equivalently, $\left(P_{1}, q_{1}\right)$. As examples, we have chosen initial preshock conditions appropriate for "cool", optically thick accretion-flows in Seyfert nuclei within a few tens of Schwarzschild radii.

Example preshock conditions:

$$
\begin{aligned}
& \text { Case 1: } n_{1}=10^{18} \mathrm{~cm}^{-3} \text { and } q_{1}=1.7 \times 10^{-5} \\
& \text { Case 2: } n_{1}=10^{20} \mathrm{~cm}^{-3} \text { and } q_{1}=1.7 \times 10^{-6}
\end{aligned}
$$

Adiabats are plotted in Figure 1. The initial preshock state is represented in the bottom right corner, and shock strength increases to the left. Each point on the adiabat is a possible postshock state, the actual state being determined by the shock speed (or Mach number).

Three regions are shown in Figure 1 that delineate the major physical processes controlling the behavior of the postshock plasma. Weak shocks remain gas-dominated with density increasing with shock strength. Moderate shocks become dominated by radiation pressure and reach a limiting density enhancement of $n_{2} / n_{1} \sim 7$. (The classical analysis, neglecting radiation, gives a density 


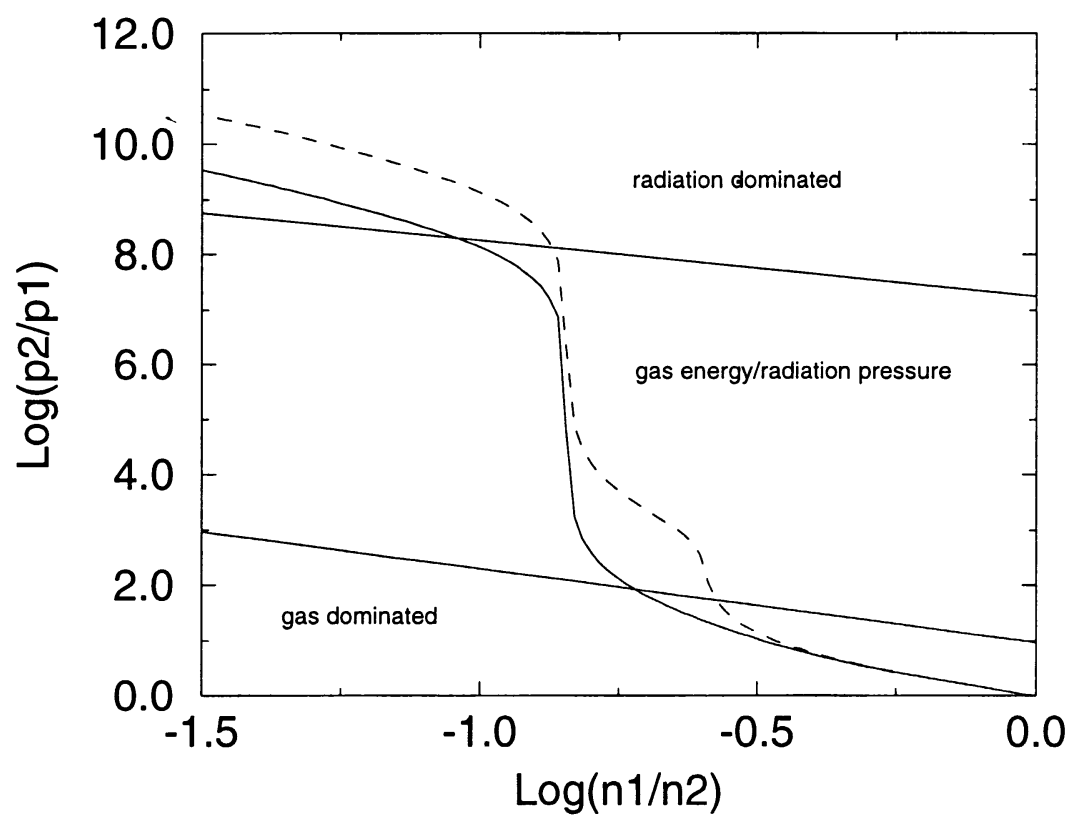

Figure 1. Shock adiabatic for case 1 (solid curve) and case 2 (dashed curve). Preshock conditions are at lower right with shock strength increasing to the left. Solid lines divide the figure into gas-dominated, gas-energy/radiation-pressure, and radiation-dominated regions.

limit of $n_{2} / n_{1} \sim 4$.) Finally, when radiation energy dominates, the density can increase without bound. Relativistic effects allow for very large density and temperature enhancements across the shock front.

\subsection{Postshock Dynamics}

Heated and over-dense postshock material will return to the equilibrium state through thermalization with the surrounding plasma or through dynamical effects such as expansion and radiative cooling. Shocks larger than the thermalization length, $\lambda_{t h} \sim \sqrt{l h}$, where $l$ is the particle deflection length and $h$ is the plasma scale height, will exist over dynamical timescales and may be seen as pockets of dense, high-temperature plasma. If these pockets become optically thin, they may have observational consequences, contributing to the X-ray spectra. Setting the effective optical depth to unity for a given physical length scale gives a curve on the $P-(1 / n)$ plane above which the plasma is optically thin. For the smaller length scale, a moderate shock can cause the postshock material to become optically thin. In such cases, the excess shock energy will likely be lost in a burst of high-energy radiation. For larger postshock length scales, the moderately shocked plasma will likely undergo adiabatic expansion and plasma becomes optically thin in this case, as well. A careful analysis suggests that an 
optically thin phase is highly likely for postshock plasma regions larger than the thermalization length (Caditz \& Tsuruta 1998).

\section{References}

Caditz, D. M., \& Tsuruta, S. 1998, ApJ, 501, 242

Iwamoto, N. 1989, Phys. Rev. A, 39, 4076

Svensson, R. 1984, MNRAS, 209, 175 